\title{
A novel Micropump Driver used in environmental sensor applications
}

\author{
Bernadette Kinzel $^{* \circ}$, Detlef Bonfert", Florian Lippert*, Frank Vanselow*, Erkan Isa*, Doris Schmitt-Landsiedel ${ }^{+}$, \\ Linus Maurer ${ }^{*}$ \\ *Fraunhofer Research Institution for Microsystems and Solid State Technologies EMFT, Munich, Germany \\ ${ }^{+}$Technical University Munich, Department of Technical Electronics, Munich, Germany \\ ${ }^{\circ}$ University of Bundeswehr, Department of Circuit Design, Neubiberg, Germany \\ Email: bernadette.kinzel@emft.fraunhofer.de
}

\begin{abstract}
The reaction times of environmental sensor units are potentially improved by the use of micropumps. Those micropumps require miniaturized driver electronics which generate dual-polarity high voltage pulses optimized to drive light, capacitive load piezoelectric micropumps. This paper presents a novel micropump driver consisting of a DC/DC converter circuit that is based on a combination of a boost converter and a charge pump. This combination generates asymmetric high voltages on two individual synchronous output nodes from a 5-V supply. Through reduction in the number of coils this method supports the overall system shrinking and allows a high level of chip integration. Compared to state-of-theart miniaturized drivers, the presented topology allows singleended and differential micropump driving at higher voltage levels $(-100 \mathrm{~V} /+150 \mathrm{~V})$. By connecting the micropump differential to the converter output, the maximum voltage can be increased to $250 \mathrm{~V}$ for capacitive micropump loads of up to $200 \mathrm{nF}$. The converter topology was validated by measurement. The presented novel, miniaturized micropump driver topology allows micropump integration into mobile devices for new fields of sensor applications with rapid reaction times.
\end{abstract}

Keywords- DC/DC Converter, Micropump Driver, LowPower Loads, Piezoelectric Driver

\section{INTRODUCTION}

Current state of the art micropump designs are manufactured on silicon and based on microelectromechanical systems (MEMS) which are controlled by a piezoelectric actuator. They are typically $5 \mathrm{~mm}$ x $5 \mathrm{~mm}$, allowing a stroke rate for liquid of $50 \mathrm{nl}$ at a max. back pressure air of $30 \mathrm{kPa}$, and are supporting a flow rate of $300 \mu \mathrm{l} / \mathrm{min}$ [1]. These micropumps are aimed for applications in the mobile environmental gas detection domain where high sensitivity, fast detection speed and system size play a major role. In applications where environmental sensors are built-in mobile devices, the ambient air has to overcome a certain diffusion path that strongly limits the sensor reaction time up to a few minutes [2]. As shown in Fig.1, independent from the diffusion path, the sensor reaction time can further be decreased, by magnitudes of up to 40 through generating an air stream for transporting the environmental change faster to the sensor. Sensor tests with a typical integrated environmental unit like Bosch BME280, in a climate chamber

This work has been supported through European H2020 ADMONT project. EU JU grant agreement $n^{\circ} 661796$

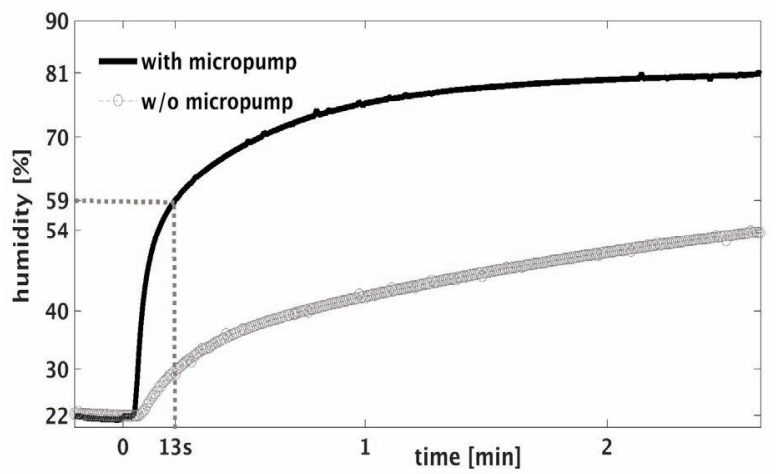

Fig.1. BME280 humidity sensing built-in smartphone measured reaction time with and without micropump

at room temperature conditions and a diffusion path, between smartphone housing and sensing layer of $10 \mathrm{~mm}$ showed improved humidity sensor reaction times of 13 seconds ( $\Delta \tau=63 \%$ ) by the use of a micropump compared to 8 minutes sensor reaction time $(\Delta \tau=63 \%)$ without additional improvement techniques (eg. software, micropump). Miniaturized micropump designs for mobile applications (pump smaller than $7 \mathrm{~mm} \times 7 \mathrm{~mm}$ ) with piezo-electric actuator layer thicknesses below $100 \mu \mathrm{m}$ require miniaturized driver electronics that are able to generate high voltage waveforms of dual polarity with maxima of $-70 \mathrm{~V} /+140 \mathrm{~V}$ in a quasi-sinusoidal waveform [1]. The quasi-sinusoidal shape of the voltage waveforms ensures reliable displacement of the piezoelectric actuator avoiding oscillation effects and therefore good pumping accuracy and reliability. To be able to use micropumps also for safety critical applications, where undefined voltage potentials lead to certain risks, the driver requires synchronous high voltage generation (positive and negative) to be able to connect the micropump single-ended as well as differential. Single-ended connection allows safe operation because of the always grounded micropump. Differential connection allows higher voltage levels due to the micropump connection between positive and negative high voltage node.

In this paper a novel DC-DC converter topology for dual polarity high-voltage generation integrated into a micropump 
driver is proposed, which fulfills the required characteristics for driving piezoelectric actuators. The proposed converter contains only one external inductor and generates the positive and negative polarity high-voltages synchronous.

\section{DUAL-POLARITY-BOOST-PUMP}

\section{A. Proposed Circuit}

The proposed DC/DC converter is depicted in Fig.2 [3] and integrated in the micropump driver system from Fig.3. The elements $\mathrm{L}, \mathrm{D}_{1}$ and $\mathrm{M}_{1}$ form a Boost Converter that drives a Dickson Charge Pump with a positive high voltage signal $\mathrm{V}_{1}$ constituted by elements $C, D_{2}$ and $D_{3}$. The two converter output nodes ( $\mathrm{V}_{\text {out }}$ and $\mathrm{V}_{\text {out-) }}$ are connected to the micropump either alternately with connection to ground (when the switches $1 \& 3$ or $2 \& 3$ are closed) or differential (when the switches $1 \& 2$ are closed), as displayed in Fig.4 and represented as high voltage multiplexer (HV MUX) in Fig.3. The output voltages ( $\mathrm{V}_{\text {out }}$ and $\mathrm{V}_{\text {out-) }}$ are sensed individually through a resistor divider network that is connected to the nonzero $\mathrm{V}_{\mathrm{ss}}$ voltage node. This sensing technique forces the sensing nodes $\mathrm{V}_{\mathrm{s} 1}$ and $\mathrm{V}_{\mathrm{s} 2}$ to an always positive voltage level even when negative voltage appears at the micropump. The PWM CTRL Block generates a modulated CLK signal $\left(=\mathrm{V}_{\mathrm{g}}\right)$, taking both sensed control signals, a input clk signal with fixed frequency and an externally applied startup signal into account to limit the output voltages to a minimum/maximum of $70 \mathrm{~V} /+140 \mathrm{~V}$. The circuit is studied on a $0.35-\mu \mathrm{m}$ HighVoltage (HV) Bulk CMOS process from XFAB.

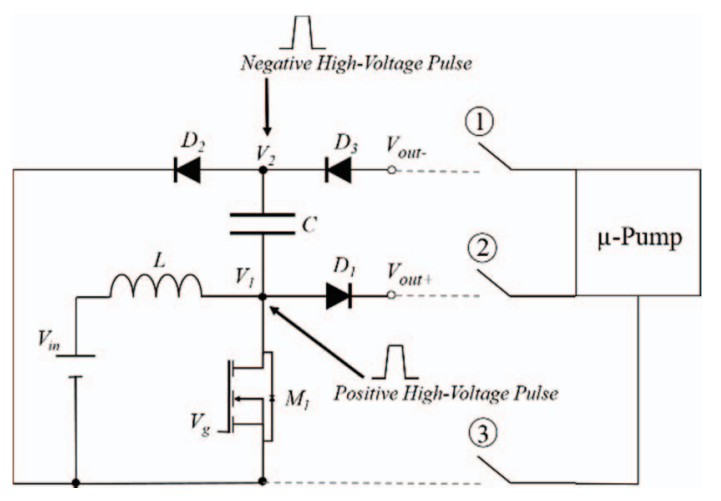

Fig.2. Structure of proposed DC/DC converter

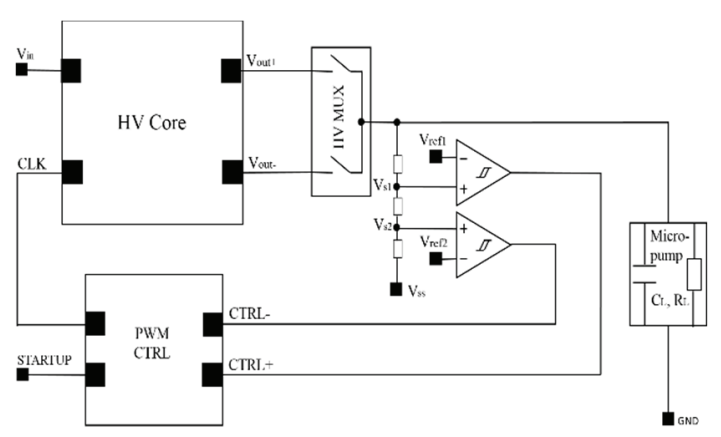

Fig. 3. Micropump Driver System consisting of the proposed DC/DC converter (HV Core), a High Voltage Multiplexer (HV MUX), Comparators and the Pulse Frequency Modulation Control (PWM CTRL) Block with Startup Input Signal

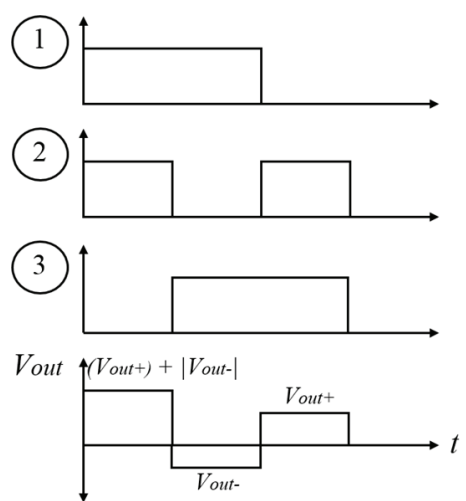

Fig. 4. Output waveform for different switch combinations of switches 1, 2 and 3

\section{B. Operating Principle}

The proposed DC/DC converter in Fig.2 is dominated in its operating principle by the traditional Boost Converter [4]. For the sake of explanation simplicity, the voltages across the switch and diodes are neglected. The power flow and highvoltage pulse generation for the transistor M1, ON-state $\left(\mathrm{V}_{\mathrm{g}}=\right.$ high $)$ and transistor OFF-state $\left(\mathrm{V}_{\mathrm{g}}=\right.$ low $)$, are explained for discontinuous conduction mode (DCM).

During the transistor $\mathrm{M}_{1} \mathrm{ON}$-state $\left(\mathrm{t}_{0} \leq \mathrm{t} \leq \mathrm{t}_{1}\right)$ displayed in Fig.5, the diodes $D_{1}$ and $D_{2}$ are reverse biased while $D_{3}$ is forward biased. A path between inductor $\mathrm{L}$ and negative voltage output ( $\mathrm{V}_{\text {out-) }}$ is formed that leads to negative high voltage pulse generation on node $\mathrm{V}_{2}$ across the coupling capacitor C. During the transistor $\mathrm{M}_{1}$ OFF-state $\left(\mathrm{t}_{1} \leq \mathrm{t} \leq \mathrm{t}_{2}\right)$ the diodes $D_{1}$ and $D_{2}$ are forward biased and $D_{3}$ is reverse biased. A path between inductor and positive voltage output $\left(\mathrm{V}_{\text {out }}+\right.$ is formed that leads to positive high voltage pulse generation on node $V_{1}$ while $V_{2}$ is going to ground potential. For differential connection to the micropump (switches $1 \& 2$ closed, switch 3 open), the overall output voltage reaches a maximum value of

$$
\mathrm{V}_{\text {out }} \approx \mathrm{V}_{\text {out }+}+\left|\mathrm{V}_{\text {out }}\right| \text {, }
$$

where $\mathrm{V}_{\text {out }}$ represents the positive high voltage node that behaves equivalent to the traditional Boost Converter [4]. The signal $\mathrm{V}_{\text {out- }}$ represents the negative high voltage node that acts
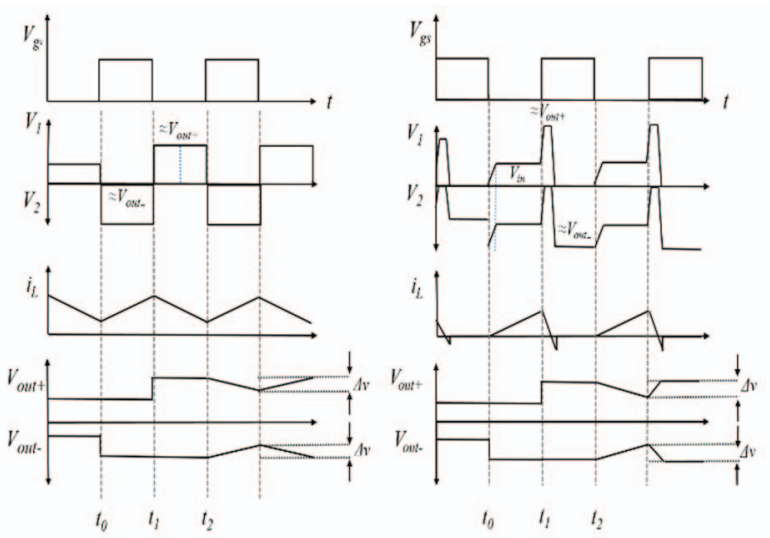

Fig.5. Key waveforms of proposed converter from Fig.2 in Continuous Conduction Mode (a) and Discontinuous Conduction Mode (b) 
like a Dickson charge pump driven by a high voltage source [5]. The converter operating mode is determined by the load conditions [4]. Micropumps are considered as capacitive load. Drivers supporting micropumps need to drive voltages up to $140 \mathrm{~V}$ at load currents in the range of $10 \mu \mathrm{A}$ - to $1 \mathrm{~mA}$. Due to concomitant needs for high gain and low output power (high output voltage but low load current), micropump drivers based on Boost Converter principles are always operated in DCM. The key waveforms of the proposed converter are displayed in Fig.5 for Continuous Conduction Mode (CCM) (a) and Discontinuous Conduction Mode (DCM) (b).

\section{RESULTS}

The proposed DC/DC converter was designed in Cadence ADE with XU035 PDK and silicon validated by measurement using Tektronix TDS7104 Oscilloscope. For the measurements, the circuit from Fig.2 was built-up with discrete diodes, capacitors and inductor. The PowerFET was integrated in XU035. The parameter values were set to: $\mathrm{L}=100 \mu \mathrm{H}, \mathrm{C}=200 \mathrm{pF}, \mathrm{f}=7.4 \mathrm{kHz}, \mathrm{V}_{\text {in }}=5 \mathrm{~V}, \mathrm{I}_{\mathrm{LOAD}}=15 \mu \mathrm{A}$ - to $3 \mathrm{~mA}$. The duty cycle of $\mathrm{f}$ was set to $50 \%$ in DCM. The measured maximum differential output voltage $\mathrm{V}_{\text {out }}$ is shown in Fig.6. The measurement results confirm that the differential output voltage can be increased to around $250 \mathrm{~V}$ for the Boost Pump by sourcing a Dickson charge pump with a Boost Converter. Under ideal conditions (not taking regulation losses into account) the power efficiency can reach a maximum percentage of around $35 \%$ for light loads below $30 \mathrm{uA}$ average load current during pump operation. The main power loss contributors in this topology are the switching losses. They are caused by the operating mode DCM that has a phase during the clk period that is neither used for energy storage nor transfer. The large area PowerFET and diodes have large parasitic capacitance that strongly limit the efficiency. The measured output voltage ripple $(\Delta v)$ is shown in Fig.7. It is defined to be the maximum output voltage deviation from $150 \mathrm{~V} /-100 \mathrm{~V}$ during steady state. The dominant circuit part for the shown $\Delta \mathrm{v}$ is the Boost Converter. The maximum ripple is defined by the inductor value, switching frequency, the resulting extrema $(\min / \max )$ of the inductor current and the output current defined by the output load impedance consisting of load capacitor and resistor [4]. The displayed Fig.7 gives the dependency between micropump size that is equivalent to a load capacitor and the output voltage ripple. The maximum $\Delta \mathrm{v}$ lies below $4 \%$ for capacitive loads up to $200 \mathrm{nF}$. Micropumps require a $\Delta \mathrm{v}$ below $10 \%$ to ensure reliable pump operation. Higher ripple percentage could lead to undesirable pumping effects.

\section{CONCLUSION}

The main benefits of the new, proposed miniaturized micropump driver compared to state-of-the-art miniaturized micropump drivers is the high connectivity flexibility that the system allows [6]. The driver can be connected single ended for safety critical applications that require one grounded load terminal. It can be connected differential for high voltage needs of up to $250 \mathrm{~V}$ taking safety margins into account. Furthermore, the output voltages $\mathrm{V}_{\text {out }}$ and $\mathrm{V}_{\text {out- }}$ are generated

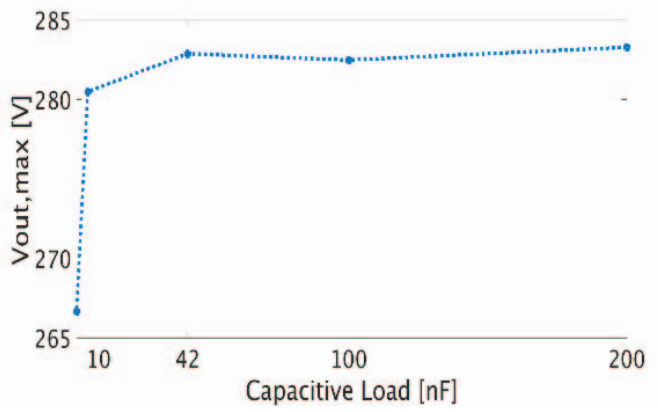

Fig.6. Maximum differential output voltage $\left(\mathrm{V}_{\text {out }}\right)$ measurement result for different capacitive loads

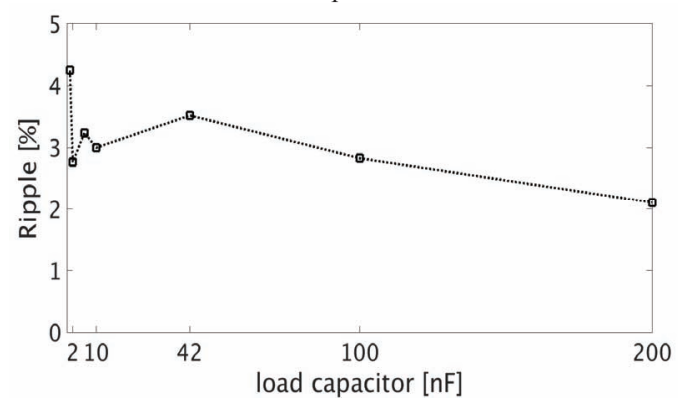

Fig.7. Output voltage ripple $(\Delta v)$ measurement result, for different capacitive loads

asymmetrically which suits to the micropump requirements and there is the need of only one external inductor. At present we are working on the full electronics integration into a single ASIC. We expect improved performance in terms of power efficiency, area and ripple through the integration. The miniaturized driver electronics allows integrating micropump-sensor modules into mobile devices where they can be used to improve the sensor reaction times at fast changing environmental conditions.

\section{ACKNOWLEDGEMENT}

We want to thank our project partners at XFAB for supporting us with the $0.35-\mu \mathrm{m}$ high-voltage technology XU035 as well as the department of micromechanics, actuators and fluidics at Fraunhofer EMFT for their support in the field of micropumps.

\section{REFERENCES}

[1] M.Herz et. al., "Method for manufacturing a bending transducer, a micro pump and a micro valve," U.S. Patent 13/604 294, Sept. 05, 2012.

[2] Seiyama, Tetsuro: Chemical Sensor Technology. Amsterdam: Elsevier, 2013

[3] B.Kinzel et al., "An integrated dual-polarity high-voltage driver concept for micropump applications," Smart System Integration Conference, March 2016.

[4] Erickson, Erickson: Fundamentals of Power Electronics. Berlin Heidelberg: Springer Science \& Business Media, 2013.

[5] Pan, Feng ; Samaddar, Tapan: Charge Pump Circuit Design. 1. Aufl.. Madison: McGraw Hill Professional, 2010.

[6] Y.K. Yong and A.J.Fleming, "Piezoelectric Actuators With Integrated High-Voltage Power Electronics," in IEEE/ASME Transactions on Meachatronics, vol. 20, no. 2, pp. 611-617, April 2015 